\title{
Prognostic microRNAs and their potential molecular mechanism in pancreatic cancer: A study based on The Cancer Genome Atlas and bioinformatics investigation
}

\author{
LIANG LIANG $^{1 *}$, DAN-MING WEI ${ }^{2 *}$, JIAN-JUN LI ${ }^{1}$, DIAN-ZHONG LUO ${ }^{2}$, \\ GANG CHEN $^{2}$, YI-WU DANG ${ }^{2}$ and XIAO-YONG CAI ${ }^{1}$
}

\begin{abstract}
${ }^{1}$ Department of General Surgery, Second Affiliated Hospital of Guangxi Medical University; ${ }^{2}$ Department of Pathology, First Affiliated Hospital of Guangxi Medical University, Nanning, Guangxi Zhuang Autonomous Region 530021, P.R. China
\end{abstract}

Received March 15, 2017; Accepted October 2, 2017

DOI: $10.3892 / \mathrm{mmr} .2017 .7945$

\begin{abstract}
Although certain biomarkers that are directly associated with the overall survival (OS) of patients with pancreatic adenocarcinoma (PAAD) have been identified, the efficacy of a single factor is limited to predicting the prognosis. The aim of the present study was to identify a combination micro (mi)RNA signature that enhanced the prognostic prediction for PAAD. Following analysis of the data available from The Cancer Genome Atlas (TCGA), 175 PAAD samples were selected for the present study, and the associations between 494 miRNAs and OS were investigated. The prognostic value of all miRNAs was analyzed by multivariate Cox regression, and the miRNAs were ranked according to the hazard ratio (HR) and P-values. The top 5 miRNAs (miR-1301, miR-125a, miR-376c, miR-328 and miR-376b) were significantly associated with OS (HR=0.139; 95\% confidence interval, 0.043-0.443; $\mathrm{P}<0.001$ ), thus demonstrating that this panel was able to serve as an independent prognostic factor for PAAD. In addition, the present study also predicted the target genes of the top 10 miRNAs with the highest prognostic values using 12 different prediction software, and enrichment signaling pathway analyses elucidated that several pathways may be markedly associated
\end{abstract}

Correspondence to: Dr Yi-Wu Dang, Department of Pathology, First Affiliated Hospital of Guangxi Medical University, 6 Shuangyong Road, Nanning, Guangxi Zhuang Autonomous Region 530021, P.R. China

E-mail: dangyiwu@126.com

Professor Xiao-Yong Cai, Department of General Surgery, Second Affiliated Hospital of Guangxi Medical University, 166 Daxue Road East, Nanning, Guangxi Zhuang Autonomous Region 530021, P.R. China

E-mail: cxy0771@163.com

*Contributed equally

Key words: pancreatic ductal adenocarcinoma, overall survival, microRNA, The Cancer Genome Atlas, pathways with these miRNAs, including 'Pathways in cancer', 'Chronic myeloid leukemia', 'Glioma' and 'MicroRNAs in cancer'. Lastly, ubiquitin C, epidermal growth factor receptor, estrogen receptor 1 , mitogen-activated protein kinase 1 , mothers against decapentaplegic homolog 4 and androgen receptor may be the hub genes revealed by STRING analysis. The present study identified several miRNAs, particularly a five-miRNA-pool, that may be reliable, independent factors for predicting survival in patients with PAAD. However, the underlying molecular mechanisms require further investigation in the future.

\section{Introduction}

Pancreatic cancer is one of the most life-threatening malignancies, accounting for more than 200,000 annual mortalities worldwide (1). It is one of the most hard-to-diagnose and aggressive malignancies, despite the increased understanding of its etiopathogenesis (2-4). Due to its fatal and asymptomatic nature, pancreatic cancer has become one of the top causes of cancer-associated mortality $(5,6)$. Among the different classes of pancreatic cancers, based on its histology, pancreatic ductal adenocarcinoma is the most predominant type, accounting for $\sim 85 \%$ of exocrine pancreatic cancers; the remainder are accounted for by papillary carcinoma and cystadenocarcinoma (7). The appropriate prediction markers are essential for clinical application; however, the performance of a single independent factor is limited in terms of accurate prediction, which, has promoted the use of combinatorial markers in clinical practice. Recent advancement in gene expression profiling technology, such as the high throughput detection method, enables researchers to identify gene-based biomarkers for PAAD diagnosis and prognosis (8). However, there are still no specific targets for the diagnosis and prognosis prediction of PAAD (9). To improve strategies applied for improving the survival of patients with PAAD, it is of great importance to identify more effective biomarkers for the diagnosis, prognosis prediction and therapeutic response of PAAD.

To date, microRNA (miRNAs) have been proposed as promising prognostic predictors for different types of cancers. miRNAs are small size RNAs, with 18-25 nucleotides, that belong to the family of non-coding RNAs (10). miRNAs have 
been reported to be involved in cell differentiation, apoptosis, proliferation and metabolism by binding to the 3 '-untranslated region of their target mRNAs, resulting in mRNA degradation and/or inhibition of mRNA translation (11). Furthermore, the associations between miRNAs and malignancies have been investigated in depth in recent years and it has been suggested that miRNAs may serve potential roles as oncogenes or tumor suppressive genes in various cancer pathogeneses (12), which, has inspired further research in to their clinical value. Previous studies have demonstrated that miRNAs are extremely stable in plasma (13), formalin-fixed paraffin-embedded tissues, and in fresh and frozen tissues $(14,15)$. Thus, miRNAs have the potential to serve as novel biomarkers for cancer diagnosis and prognosis prediction, as they remain largely intact. Several miRNAs have been reported to serve vital roles in the tumorigenesis and prognosis of PAAD (16-21). However, the associations between other miRNAs and the survival of patients with PAAD remains unclear. In addition, an miRNA profile based on The Cancer Genome Atlas (TCGA) data has not been published. Therefore, the present study analyzed the associated TCGA data, evaluating a large number of miRNA sequencing information for patients with PAAD in order to systematically assess the predictive value of a specific miRNA signature for overall survival in patients with PAAD (22).

\section{Materials and methods}

Patient characteristics and miRNA dataset in TCGA. The level 3 data of miRNA sequencing and the corresponding clinical data for 175 patients with PAAD were obtained from TCGA (tcga-data.nci.nih.gov/doc/publications/tcga/?) in July $2016(23,24)$. As all data are publicly available and open-access, ethical approval was not necessary for the present study. Clinical characteristics of PAAD patients are presented in Table I.

Survival analysis. A multivariate model was constructed with miRNA expression, sex,age,gradeandTumor-Nodes-Metastasis stage. Multivariate Cox regression was performed to evaluate the associations between the expression of all miRNAs and overall survival (OS). Hazard ratio (HR) and 95\% confidence intervals $(\mathrm{CI})$ were also assessed. The top 10 miRNAs, those that exhibited the most significant prognostic values, were selected for further analysis. The median expression of each miRNA was set as cutoff value. Once miRNAs were grouped into those with high or low expression according to its median value (Table II), Kaplan-Meier (K-M) survival curves were also generated. A permutation and combination method was adopted for the present study; it is considered overexpressed when all selected miRNAs presented overexpression simultaneously. Multivariate cox regression and Kaplan-Meier survival curves were conducted with SPSS version 22.0 software (IBM Corp., Armonk, NY, USA). The survival time of each miRNA were presented as the mean \pm standard error. $\mathrm{P}<0.05$ was considered to indicate a statistically significant difference.

Target prediction for miRNAs and functional enrichment analysis. The prospective target genes of miRNAs were predicted using 12 different programs including miRWalk2.0,
Table I. Clinical characteristics of patients with pancreatic cancer based on the The Cancer Genome Atlas.

\begin{tabular}{lr}
\hline Characteristics & Number (\%) \\
\hline Age (years) & \\
$\geq 60$ & $119(68.0)$ \\
$<60$ & $56(32.0)$ \\
Sex & \\
Female & $79(45.1)$ \\
Male & $96(54.9)$ \\
Stage & \\
I+II & $164(95.9)$ \\
III+IV & $7(4.1)$ \\
Grade & \\
G1+G2 & $125(71.4)$ \\
G3+G4 & $50(28.6)$ \\
Neoplasm status & \\
With tumor & $100(64.1)$ \\
Tumor free & $56(35.9)$ \\
Chronic pancreatitis & \\
Yes & $14(10.1)$ \\
No & $125(89.9)$ \\
Diabetes & \\
Yes & $37(25.7)$ \\
No & $107(74.3)$ \\
Alcohol history & \\
Yes & $99(61.1)$ \\
No & $63(38.9)$ \\
\hline
\end{tabular}

DIANA-microTv4.0, miRanda-rel2010, mirBridge, miRDB4.0, miRmap, miRNAMap, doRiNA i.e.,PicTar2, PITA, RNA22v2, RNAhybrid2.1 and Targetscan6.2 (25).

The overlapping genes were processed using the functional enrichment analyses, Gene Ontology (GO) enrichment, Kyoto Encyclopedia of Genes and Genomes (KEGG) and protein-protein interaction (PPI) (26). Among these approaches, $\mathrm{GO}$ is an international standardized gene functional classification system, offering a dynamic-updated controlled vocabulary and a strictly defined concept to comprehensively define properties of genes and their products in any organism. $\mathrm{GO}$ analysis reveals the cataloging of biological process (BP), cellular component (CC) and molecular function (MF). The GO enrichment study was performed using OmicShare tools, a free online platform for data analysis (www.omicshare.com/). Firstly, all target genes were mapped to GO terms in the Gene Ontology database (www.geneontology.org/) and gene numbers were calculated for each term. Significantly enriched GO terms in target genes, as compared with the genome background, were defined by a hypergeometric test. GO terms that met these criteria were defined as significantly enriched GO terms in target genes. This examination identified the key biological functions associated with the target genes.

KEGG is the major public pathway-associated database, which identifies significantly enriched metabolic pathways or 
Table II. Prognostic role of the 10 most valuable microRNAs in pancreatic cancer based on The Cancer Genome Atlas.

\begin{tabular}{|c|c|c|c|c|c|c|c|}
\hline \multirow[b]{3}{*}{ miRNA } & \multicolumn{4}{|c|}{ Kaplan-meier } & \multirow{2}{*}{\multicolumn{3}{|c|}{ Multivariate cox regression }} \\
\hline & \multirow{2}{*}{$\begin{array}{l}\text { Cutoff } \\
\text { value }\end{array}$} & \multirow{2}{*}{$\begin{array}{l}\text { High expression } \\
\text { (months) }\end{array}$} & \multirow{2}{*}{$\begin{array}{l}\text { Low expression } \\
\text { (months) }\end{array}$} & \multirow{2}{*}{$\begin{array}{l}\text { Log rank } \\
\text { P-value }\end{array}$} & & & \\
\hline & & & & & HR & $95 \% \mathrm{CI}$ & P-value \\
\hline miR-1301 & 4.80 & $45.62 \pm 4.96$ & $24.27 \pm 3.63$ & $<0.001$ & 0.450 & $0.293-0.692$ & $<0.001$ \\
\hline $\operatorname{miR}-125 \mathrm{a}$ & 650.29 & $45.97 \pm 5.00$ & $21.82 \pm 1.94$ & 0.001 & 0.484 & $0.314-0.747$ & 0.001 \\
\hline miR-376c & 0.78 & $40.67 \pm 4.01$ & $28.54 \pm 3.97$ & 0.001 & 0.499 & $0.327-0.761$ & 0.001 \\
\hline miR-328 & 22.85 & $46.01 \pm 5.08$ & $24.21 \pm 3.16$ & 0.002 & 0.517 & $0.339-0.788$ & 0.002 \\
\hline miR-376b & 0.76 & $39.96 \pm 3.97$ & $29.35 \pm 4.10$ & 0.002 & 0.521 & $0.343-0.794$ & 0.002 \\
\hline miR-29b & 23.32 & $41.83 \pm 4.56$ & $24.36 \pm 2.86$ & 0.002 & 0.522 & $0.340-0.800$ & 0.003 \\
\hline miR-454 & 3.97 & $45.93 \pm 5.10$ & $22.12 \pm 1.83$ & 0.003 & 0.523 & $0.339-0.806$ & 0.003 \\
\hline $\operatorname{miR}-664 a$ & 2.21 & $46.77 \pm 5.30$ & $26.51 \pm 3.14$ & 0.004 & 0.540 & $0.353-0.825$ & 0.004 \\
\hline $\operatorname{miR}-3613$ & 5.59 & $43.54 \pm 4.88$ & $22.46 \pm 1.96$ & 0.011 & 0.576 & $0.376-0.884$ & 0.012 \\
\hline miR-126 & $1,372.53$ & $37.39 \pm 3.81$ & $30.19 \pm 4.39$ & 0.030 & 0.634 & $0.418-0.960$ & 0.031 \\
\hline miRNA pool & & $65.74 \pm 5.30$ & $30.17 \pm 3.32$ & $<0.001$ & 0.139 & $0.043-0.443$ & $<0.001$ \\
\hline
\end{tabular}

High and low expression groups of each miRNA were generated according to its median value. Data are expressed as the mean \pm standard error. The miRNA pool consisted of miR-1301, miR-125a, miR376c, miR-328 and miR-376b. miRNA/miR, microRNA; HR, hazard ratio; CI, confidential interval.

signal transduction pathways in target genes when compared with the whole genome background. Pathway enrichment analysis was also performed through the OmicShare tools. Significantly enriched pathways in targets, when compared with the genome background, were defined by a hypergeometric test. Pathways meeting this criterion were regarded as significantly enriched pathways in target genes. GO and KEGG pathways were analyzed by calculating the False Discovery Rate Correction; 0.05 was applied as a threshold.

The interactions of all target genes were observed using the STRING online database (string-db.org), with the cut-off criterion of a combined score $>0.9$, and Cytoscape software version 3.4.0 (www.cytoscape.org/). Network nodes represent proteins, while edges are the protein-protein associations. The topological centralities, including the local scale (degree) and the global scale (betweenness, closeness and stress), were extensively utilized in the network analysis. Of these, degree is a simple and evident measure that defines the number of links between a node and its neighboring nodes (27). In the present study, nodes with a certain degree were proposed to be hub genes $(28,29)$. A sub-network composed of hub genes and their corresponding interactions were extracted from the target network.

Immunohistochemistry of The Human Protein Atlas (THPA). Immunohistochemistry images were downloaded from the publicly available database, THPA (www.proteinatlas.org/) (30,31). THPA, a database that includes $>5$ million images of immunohistochemically stained tissues and cells, is based on 6,122 antibodies, representing 5,011 human proteins encoded by $\sim 25 \%$ of the human genome. Data regarding the expression levels of UBC, SMAD4, MAPK1, AR, ESR1, HRAS and EGFR in PAAD tissues was obtained from THPA. The protein expression level of each gene was ranked according to whether it presented low, medium or high antibody staining.

\section{Results}

Survival analysis. In total, the present study successfully assessed 494 miRNAs associated with survival. The prognostic value of all miRNAs was analyzed by multivariate Cox regression (data not shown), and then the miRNAs were ranked according to the HR and P-values. The top 10 candidates (miR-1301, miR-125a, miR-376c, miR-328, miR-376b, miR-29b, miR-454, miR-664a, miR-3613 and miR-126) presented the most significant prognostic value (Fig. 1; Table II). Among these 10 miRNAs, the miRNA with the lowest HR was miR-126, which, had a HR of 0.634 (95\% CI: $0.418-0.96, \mathrm{P}=0.031)$, and the miRNA with the greatest prognostic value was miR-1301 ( $\mathrm{HR}=0.45$; 95\% CI, 0.293-0.692; $\mathrm{P}<0.001)$. The high and low expression groups of each miRNA were formed based on the cutoff median level, and the K-M method was performed. The patients in the high expression group containing the top 10 miRNAs had markedly better survival than those of the low expression group (Table II). For example, the average survival of the high expression miR-1301 group was $45.62 \pm 4.96$ months, a significantly longer survival period than the $24.27 \pm 3.63$ months exhibited by the low expression group $(\mathrm{P}<0.001)$. In addition, miR-1301 also had the most significant prognostic value based on K-M analysis. To produce a more effective prognostic tool for clinical practice, the present study combined the top 5 miRNAs into a pool. It was demonstrated that the miRNA pool comprised of miR-1301, miR-125a, miR-376c, miR-328 and miR-376b effectively enhanced the efficiency of prognostic prediction for survival (Fig. 2). The multivariate Cox regression produced consistent results, revealing that all 10 miRNA candidates were able to act as protective factors as their HRs were $<1$, ranging from 0.634 (miR-126) to 0.450 (miR-1301; Table II). Notably, the 5-miRNA-pool exhibited an ideal prognostic value, with a HR value of 0.139 (95\% CI, 0.043-0.443; P<0.001), which, 

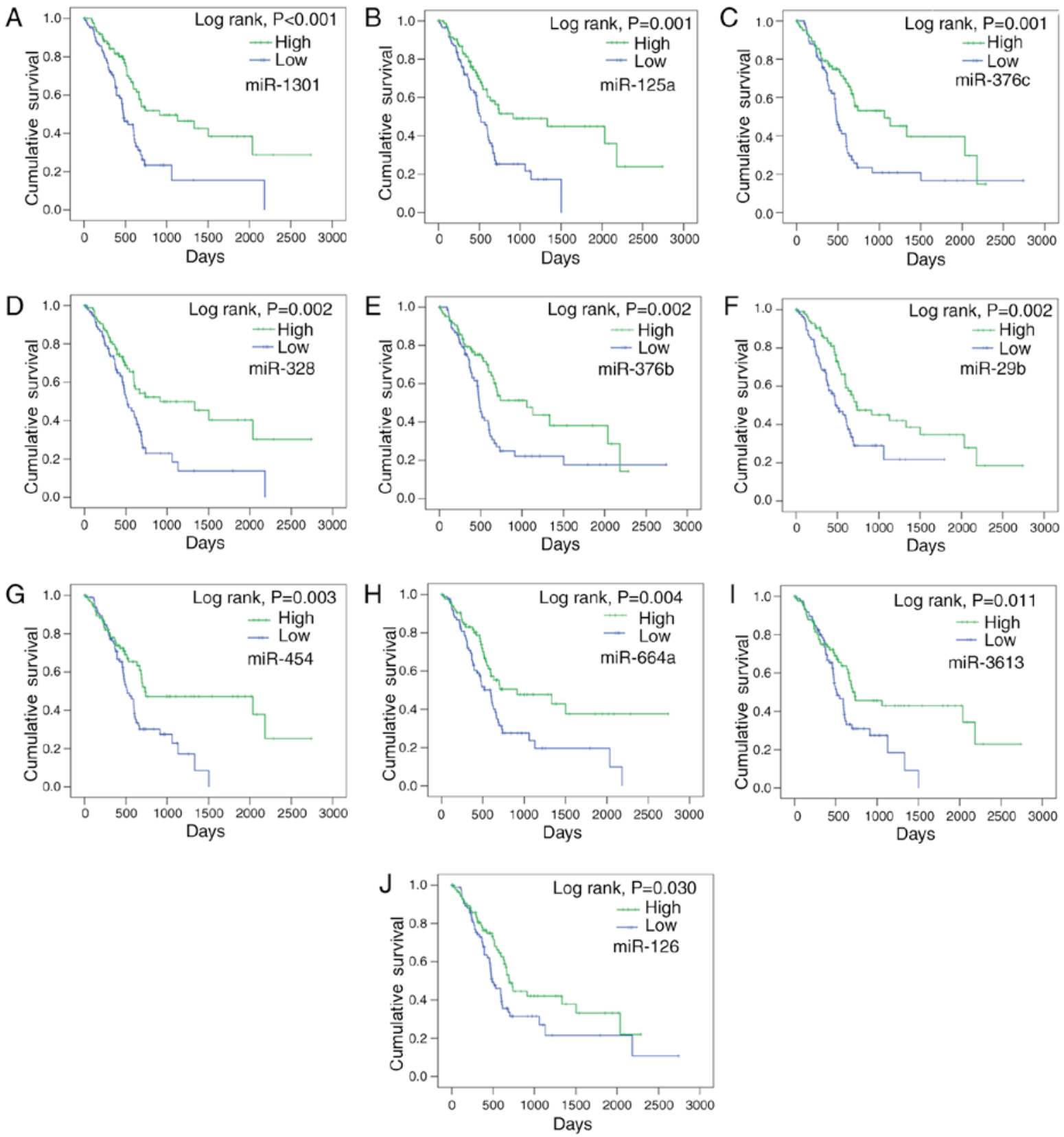

Figure 1. Associations between the levels of candidate miRNAs and survival in pancreatic cancer. The associations between the levels of the top 10 candidate miRNAs, (A) miR-1301, (B) miR-125a, (C) miR-376c, (D) miR-328, (E) miR-376b, (F) miR-29b, (G) miR-454, (H) miR-664a, (I) miR-3613 and (J) miR-126. $\mathrm{miR}$, and survival in pancreatic cancer were analyzed using the Kaplan-Meier method. miRNA/miR, microRNA.

supported the notion that this 5-miRNA-pool may have a greater predictive capacity for prognosis than individual miRNA (Table II).

Target prediction. The target genes $(\mathrm{n}=490)$ of miR-376b and miR-376c were selected when they appeared in $>5$ of the 8 programs available. The 624 target genes from the remaining 8 miRNAs (miR-1301, miR-125a, miR-328, miR-29b, miR-454, miR-664a, miR-3613 and miR-126) were selected when they appeared $>9$ times in the 12 software. The 35 overlapping genes were omitted, leaving a total of 1,079 target genes for further functional enrichment analysis (Fig. 3).

Functional enrichment analysis. Enrichment analyses were then performed to elucidate the biological function of the target genes of 10 miRNAs. GO analysis revealed that 52 signaling pathways were significantly enriched for BP, 20 for CC and 22 for MF (Fig. 4; Table III). KEGG analysis demonstrated that the top pathway in the KEGG_A_class was 'Human Diseases', and in KEGG_B_class it was 'Cancers', of which, several pathways were closely associated, including 'Pathways in cancer', 'Chronic myeloid leukemia', 'Glioma' and 'microRNAs in cancer' (Fig. 5; Table IV).

PPI network analysis of all target genes using the STRING database revealed that 854 interactions were involved, with combined scores of no less than 0.9. Networks were also constructed for miR-376b and miR-376c (Fig. 6), and the remaining 8 miRNAs (Fig. 7), as different predicting programs were utilized. Nodes with $\geq 20$ degrees were defined as hub genes, including ubiquitin $\mathrm{C}$ (UBC; $\mathrm{n}=132$ ), mothers against 


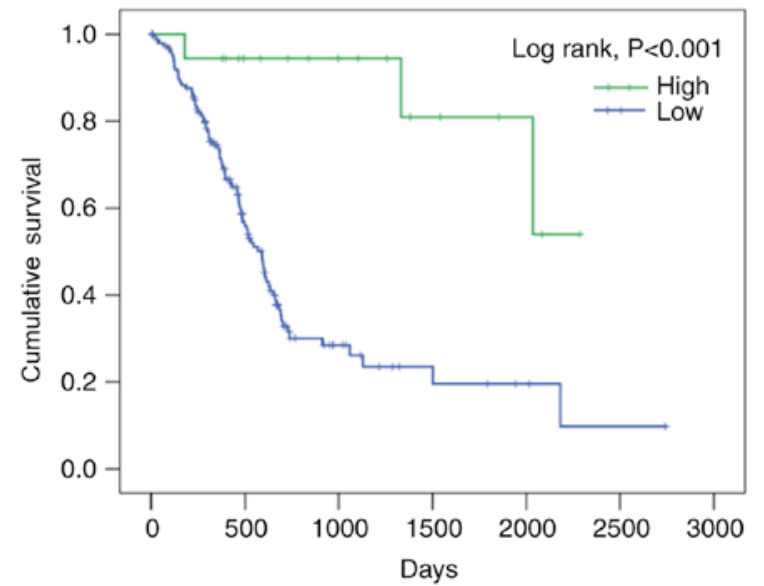

Figure 2. Combinatorial prognostic value of 5 miRNAs for pancreatic cancer. The most significant individual miRNAs $(n=5)$ were selected to form a pool and its association with survival in pancreatic cancer was analyzed using the Kaplan-Meier method. miRNA, microRNA.

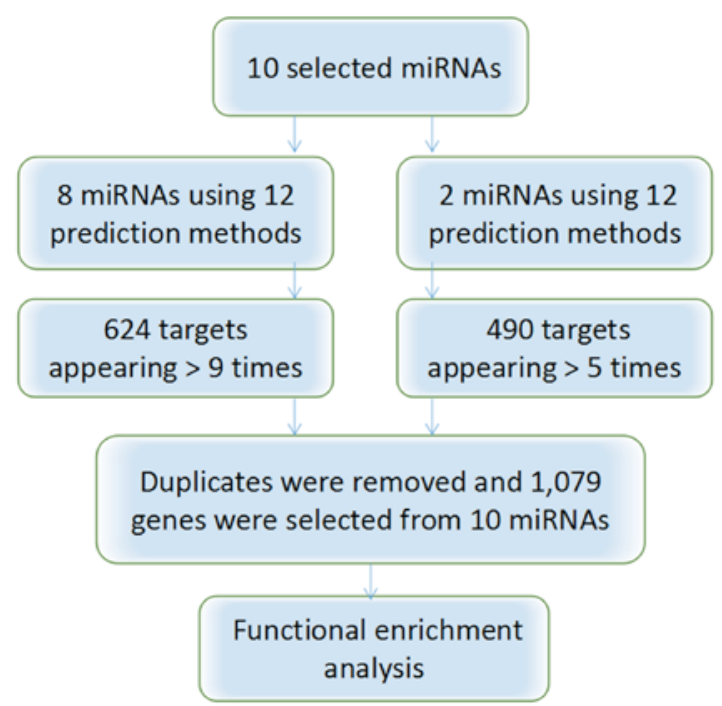

Figure 3. Flow-process diagram of the bioinformatics analysis. The process of screening utilized in the present study is presented in the diagram for the prospective targets of the 10 miRNAs. miRNA, microRNA.

decapentaplegic homolog 4 (SMAD4; n=25), mitogen-activated protein kinase 1 (MAPK1; $n=24)$, androgen receptor (AR; $\mathrm{n}=23$ ), estrogen receptor 1 (ESR1; $\mathrm{n}=21$ ), HRas proto-oncogene (HRAS; $\mathrm{n}=20$ ) and epidermal growth factor receptor (EGFR; $\mathrm{n}=20$ ). Sub-networks comprised of hub genes and their corresponding interactions were extracted from the target networks, as depicted in Fig. 8.

Validation of hub gene expression by immunochemistry. The present study examined the protein expression of the hub genes. THPA im ages revealed a markedly high expression of EGFR could be observed using the CAB000035 antibody in pancreatic cancer. In 12 patients, 2 presented high levels of staining, 7 had medium levels of staining and 3 exhibited low levels of staining; however, no EGFR expression was detected in the 3 normal counterparts (Figs. 9 and 10). The remaining protein expression data were not markedly different in the protein atlas.

\section{Discussion}

In the present study, the prognostic significance of each miRNA was calculated by multivariate cox regression analysis, and was adjusted to the influence of clinical features and factors including tumor grade, age and sex. The results identified the top 10 miRNAs with the highest prognostic value which, were selected for subsequent analysis in PAAD based on the TCGA data. These 10 miRNAs, which, may be able to serve as significant independent predictors for survival, included miR-1301, miR-125a, miR-376c, miR-328, miR-376b, miR-29b-2, miR-454, miR-664a, miR-3613 and miR-126. The miRNA expression data was then separated into high and low expression groups. The 5-miRNA-pool, which, included the top 5 miRNAs in a combined signature (miR-1301, miR-125a, miR-376c, miR-328 and miR-376b), had the greatest ability to predict the prognosis of patients with pancreatic cancer. This 5-miRNA-pool presented an ideal prognostic value with a HR of 0.139 (95\% CI, 0.043-0.443; $\mathrm{P}<0.001$ ), and may provide novel biomarkers for clinical application. The potential target genes of the aforementioned miRNAs were enriched in 'Pathways in cancer', the signaling pathways of 'Chronic myeloid leukemia' and 'Glioma'. Consequently, among the predicted targets of the relevant miRNAs for PAAD, UBC, EGFR, MAPK1, ESR1, SMAD4 and AR were considered to be hub genes in the present study.

miRNAs are gaining more attention in current research on account of their close association with tumor progression, particularly in PAAD. Previously, Ma et al (32) published an evaluated meta-review based on miRNA expression profiling studies from the Scopus database, Gene Expression Omnibus, ArrayExpress and PubMed, using the vote-counting strategy and robust rank aggregation method. The group revealed that the most significantly upregulated miRNAs included miR-155, miR-100, miR-21, miR-221, miR-31, miR-143 and miR-23a, and the frequently downregulated miRNAs from the different approaches were miR-217, miR-148a and miR-375. Notably, when evaluating the prognostic role of these aberrantly expressed miRNAs by multivariate analyses, miR-21, miR-31 and miR-375 were able to act as prognostic biomarkers for PAAD (32). Frampton et al (33) also performed a meta-analysis in 2015 in order to examine the effect of miRNAs on OS and disease-free survival in PAAD, based on 20 eligible studies with 1,525 patients and assessed via microarrays, reverse transcription-quantitative polymerase chain reaction or in situ hybridization. The group confirmed that OS was markedly shortened in patients with high tumoral miR-21. In addition, the significant miRNAs, miR-155, miR-203, miR-34a, miR-222 and miR-10b, were identified, and were thought to be potential prognostic biomarkers for PAAD (33). Thus, different miRNAs have previously been shown to have prognostic value in PAAD.

However, to the best of our knowledge, no report has been published for the prognostic role of miRNAs based on the public data from TCGA. TCGA has provided clinicians and researchers unprecedented access to information regarding various malignancies through numerous platforms, including exome sequencing, comparative genomic hybridization arrays, DNA methylation arrays, RNA sequencing and reverse protein phase arrays, as well as clinicopathological characteristics (34). 
Table III. Enriched Gene Ontology terms for the target genes of the top 10 microRNAs.

\begin{tabular}{|c|c|c|c|c|}
\hline$\#$ & Pathway ID & Pathway description & P-value & FDR \\
\hline & \multicolumn{4}{|l|}{ Biological process } \\
\hline 1 & GO:0032502 & Developmental process & $7.40 \times 10^{-13}$ & $6.95 \times 10^{-11}$ \\
\hline 2 & GO:0048856 & Anatomical structure development & $8.33 \times 10^{-13}$ & $6.95 \times 10^{-11}$ \\
\hline 3 & GO:0009653 & Anatomical structure morphogenesis & $1.96 \times 10^{-11}$ & $1.09 \times 10^{-9}$ \\
\hline 4 & GO:0044767 & Single-organism developmental process & $2.17 \times 10^{-9}$ & $9.07 \times 10^{-8}$ \\
\hline \multirow[t]{2}{*}{5} & GO:0030154 & Cell differentiation & $3.52 \times 10^{-9}$ & $1.17 \times 10^{-7}$ \\
\hline & \multicolumn{4}{|l|}{ Cellular component } \\
\hline 1 & GO:0005622 & Intracellular & $2.43 \times 10^{-12}$ & $9.20 \times 10^{-11}$ \\
\hline 2 & GO:0044424 & Intracellular part & $2.92 \times 10^{-12}$ & $9.20 \times 10^{-11}$ \\
\hline 3 & GO:0005634 & Nucleus & $7.69 \times 10^{-11}$ & $1.61 \times 10^{-9}$ \\
\hline 4 & GO:0043227 & Membrane-bounded organelle & $4.37 \times 10^{-9}$ & $5.51 \times 10^{-8}$ \\
\hline \multirow[t]{2}{*}{5} & GO:0043231 & Intracellular membrane-bounded organelle & $4.37 \times 10^{-9}$ & $5.51 \times 10^{-8}$ \\
\hline & \multicolumn{4}{|l|}{ Molecular function } \\
\hline 1 & GO:0005488 & Binding & $6.53 \times 10^{-25}$ & $4.11 \times 10^{-23}$ \\
\hline 2 & GO:0001071 & Nucleic acid binding transcription factor activity & $1.85 \times 10^{-16}$ & $5.82 \times 10^{-15}$ \\
\hline 3 & GO:0043167 & Ion binding & $1.25 \times 10^{-15}$ & $2.62 \times 10^{-14}$ \\
\hline 4 & GO:0003677 & DNA binding & $1.75 \times 10^{-15}$ & $2.75 \times 10^{-14}$ \\
\hline 5 & GO:0003676 & Nucleic acid binding & $1.02 \times 10^{-10}$ & $9.16 \times 10^{-10}$ \\
\hline
\end{tabular}

The top 5 GO terms with a lower P-value in Biological Process, Cellular Component and Molecular Function, respectively. GO, Gene Ontology; FDR, false discovery rate.

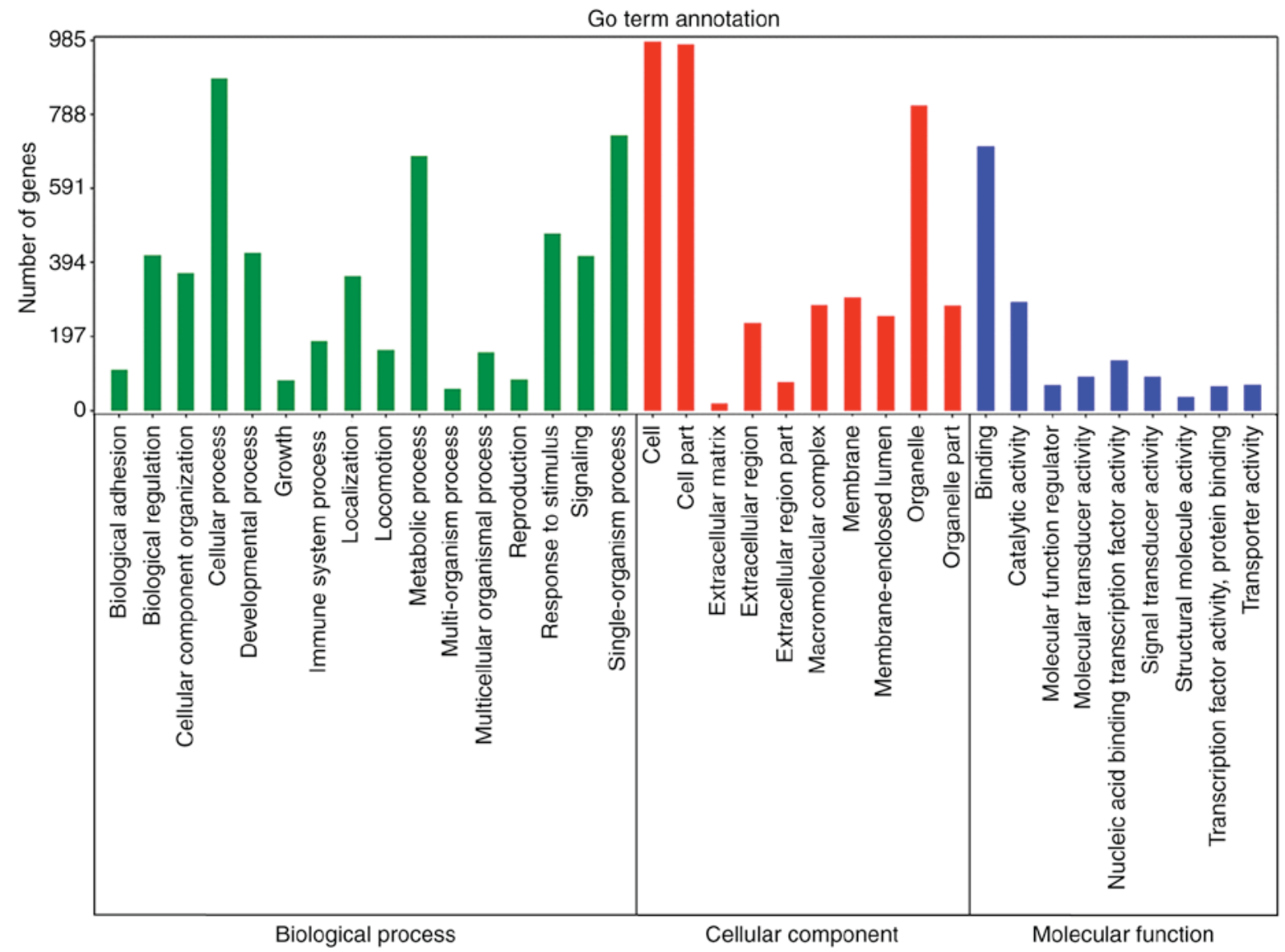

Figure 4. GO terms from the predicted target genes of the 10-microRNA signature. The target genes ( $\mathrm{n}=1,079)$ were input for GO analysis using OmicShare tools. Significant pathways were determined according to the false discovery rate and the gene number. The green bars represent pathways in the 'Biological Process', red bars represent 'Cellular Component' pathways and blue bars are the 'Molecular Function' pathways. GO, Gene Ontology. 
KEGG pathway annotation

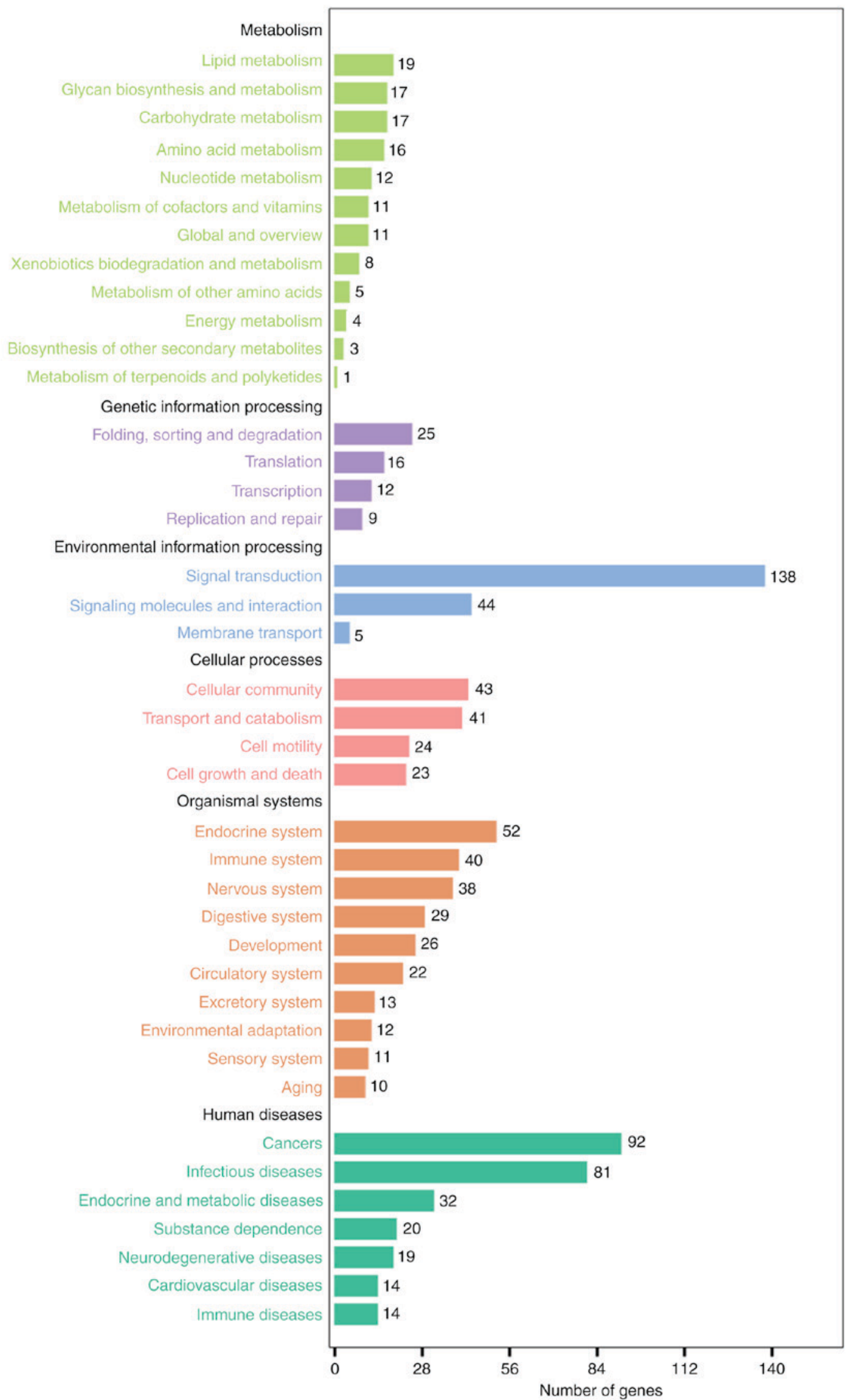

Figure 5. KEGG pathway annotation of the predicted target genes from the 10-microRNA signature. KEGG analysis using OmicShare tools was performed with the target genes $(n=1,079)$. Significant pathways were determined according to the false discovery rate and the gene number. The bars for each KEGG pathway are listed according to their molecular interactions and reaction networks within 'Metabolism' (light green bars), 'Genetic information processing' (purple bars), 'Environmental information processing' (blue bars), 'Cellular processes' (red bars), 'Organismal systems' (orange bars) and 'Human diseases' (dark green bars). KEGG, Kyoto Encyclopedia of Genes and Genomes. 
Table IV. Kyoto Encyclopedia of Genes and Genomes pathway terms for the target genes of the top 10 microRNAs.

\begin{tabular}{|c|c|c|c|c|c|}
\hline Pathway ID & KEGG_A_class & KEGG_B_class & Pathway & P-value & Q-value \\
\hline ko05200 & Human diseases & Cancers & Pathways in cancer & $4.79 \times 10^{-6}$ & $5.40 \times 10^{-4}$ \\
\hline ko05220 & Human diseases & Cancers & Chronic myeloid leukemia & $6.50 \times 10^{-6}$ & $5.40 \times 10^{-4}$ \\
\hline ko05214 & Human diseases & Cancers & Glioma & $9.60 \times 10^{-6}$ & $5.40 \times 10^{-4}$ \\
\hline ko05206 & Human diseases & Cancers & MicroRNAs in cancer & $1.04 \times 10^{-5}$ & $5.40 \times 10^{-4}$ \\
\hline ko04010 & $\begin{array}{l}\text { Environmental } \\
\text { information processing }\end{array}$ & Signal transduction & MAPK signaling pathway & $1.04 \times 10^{-5}$ & $5.40 \times 10^{-4}$ \\
\hline ko05212 & Human diseases & Cancers & Pancreatic cancer & $3.90 \times 10^{-5}$ & $1.47 \times 10^{-3}$ \\
\hline ko05205 & Human diseases & Cancers & Proteoglycans in cancer & $3.97 \times 10^{-5}$ & $1.47 \times 10^{-3}$ \\
\hline ko05215 & Human diseases & Cancers & Prostate cancer & $7.69 \times 10^{-5}$ & $2.49 \times 10^{-3}$ \\
\hline ko04540 & Cellular processes & Cellular commiunity & Gap junction & $1.11 \times 10^{-4}$ & $3.20 \times 10^{-3}$ \\
\hline ko04810 & Cellular processes & Cell motility & Regulation of actin cytoskeleton & $3.36 \times 10^{-4}$ & $8.70 \times 10^{-3}$ \\
\hline
\end{tabular}

The pathway terms listed are those with the lowest P-values. The Q-value represents the adjusted P-value. KEGG, Kyoto Encyclopedia of Genes and Genomes.

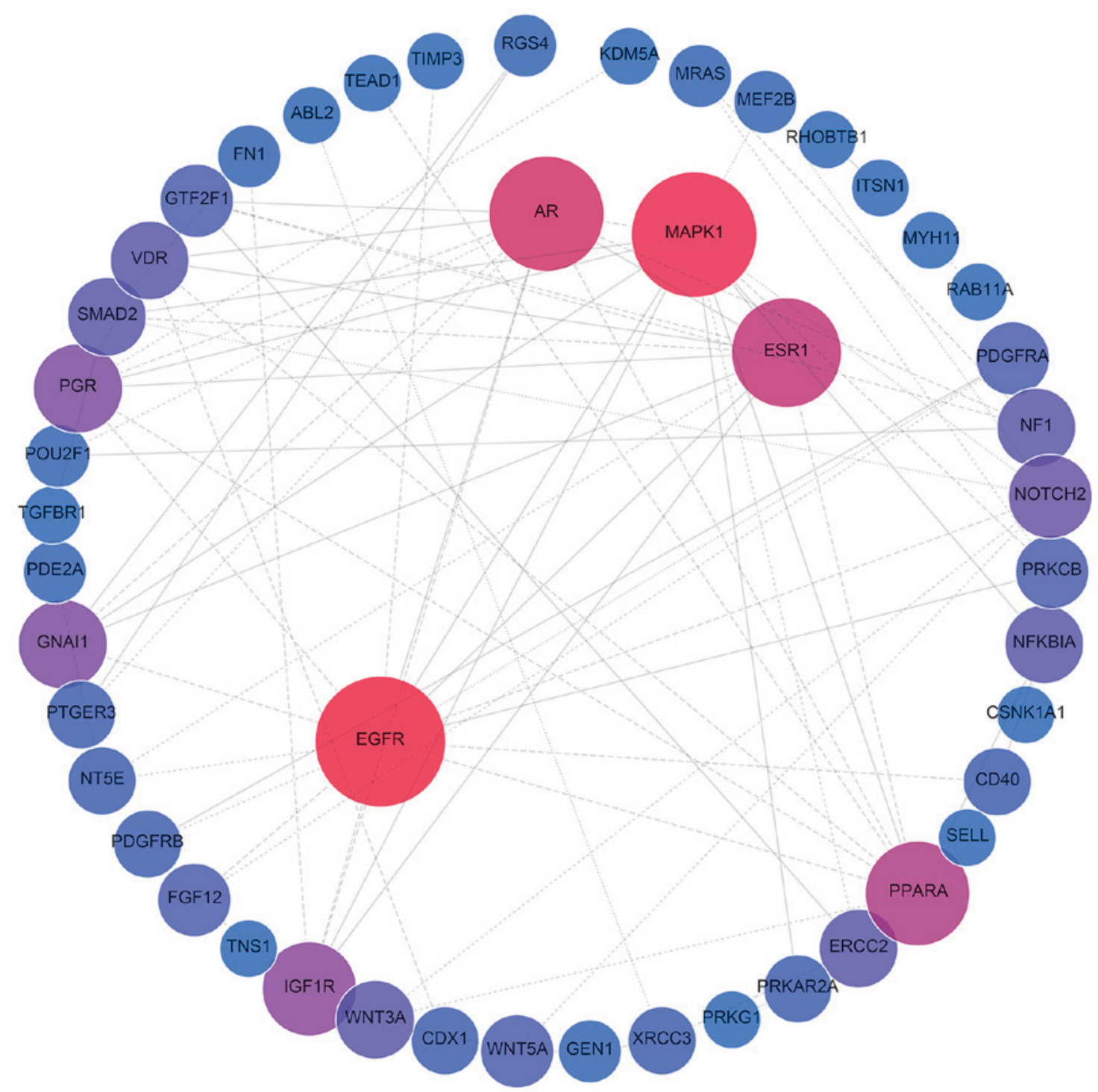

Figure 6. Protein-protein interaction network analysis of all miR-376b and miR-376c target genes. Gene interactions were constructed using the STRING online database (string-db.org) and Cytoscape (version 3.4.0; www.cytoscape.org/). Network nodes represent proteins and the edges represent protein-protein associations. The predicted target genes $(n=490)$ of miR-376b and miR-376c were analyzed here, as only 5 different software were available for prediction. miRNA/miR, microRNA. 


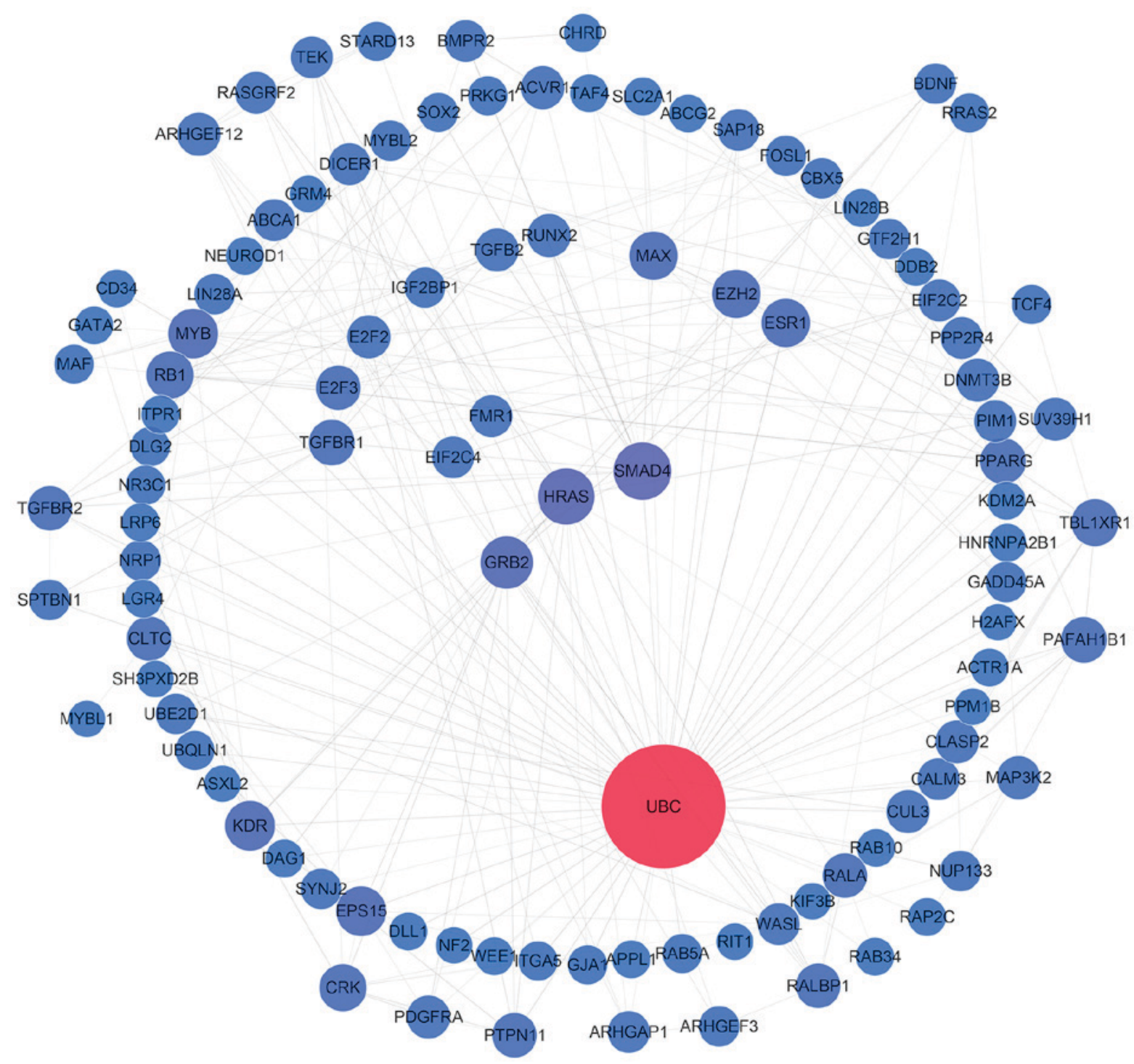

Figure 7. Protein-protein interaction network analysis of the target genes of miR-1301, miR-125a, miR-328, miR-29b, miR-454, miR-664a, miR-3613 and miR-126. Gene interactions were constructed using the STRING online database (string-db.org) and Cytoscape (version 3.4.0; www.cytoscape.org/). Network nodes represent proteins and edges represent protein-protein associations. Predicted target genes $(\mathrm{n}=624)$ of the remaining 8 miRNAs (miR-1301, miR-125a, miR-328, miR-29b, miR-454, miR-664a, miR-3613 and miR-126) were analyzed. miRNA/miR, microRNA.

The miRNA-seq TCGA data of PAAD has not been utilized for the investigation of the prognostic role of miRNAs. In the present study, the prognostic values of all miRNAs for PAAD available in TCGA were calculated, and the 10 most efficient miRNAs for prognosis prediction were revealed to be miR-1301, miR-125a, miR-376c, miR-328, miR-376b, miR-29b, miR-454, miR-664a, miR-3613, and miR-126. This appears to be a novel finding as these 10 miRNAs were not reported in the meta-analyses performed either by Ma et al (32) or Frampton et al (33). All 10 miRNAs were confirmed as protective factors, with the HR values ranging from 0.450 to 0.634 . Furthermore, among the 10 miRNAs, the 5-miRNA signature, consisting of miR-1301, miR-125a, miR-376c, miR-328 and miR-376b, were pooled as a combined predictor with a HR of 0.139 . The combination of these 5 miRNAs may provide a novel tool for clinical application in predicting the prognosis of patients with PAAD. However, this hypothesis requires further studies involving larger cohorts and multiple detection methods to confirm.
Some of the functions and molecular mechanisms associated with the 10 miRNAs selected in the present study have been reported previously. For example, miR-1301-3p has been extensively studied in some cancers. Fang et al (35) concluded that miR-1301-3p may function as an inhibitor of tumorigenesis, regulating cell apoptosis through B-cell lymphoma-2 (Bcl-2) and Bcl-xL in hepatocellular carcinoma HepG2 cells. Lin et al (36) indicated that miR-1301-3p-induced downregulation of Ran GTPase activating protein 1 may activate the p73-dependent apoptosis pathway by mediating breakpoint cluster region-Abelson tyrosine-protein kinase nuclear entrapment, which, may be a novel strategy for improving the current Imatinib treatment for chronic myeloid leukemia. Another study demonstrated that miR-1301-3p was upregulated in prostate cancer cells and tissues (37). miR-1301-3p accelerated the G1/S transition to advance cell cycle progression by impeding p27 expression and increasing Cyclin D1 expression (37). This study also revealed that miR-1301-3p targeted protein phosphatase 2 regulatory subunit $\mathrm{Bg}$, further suppressing tumor 


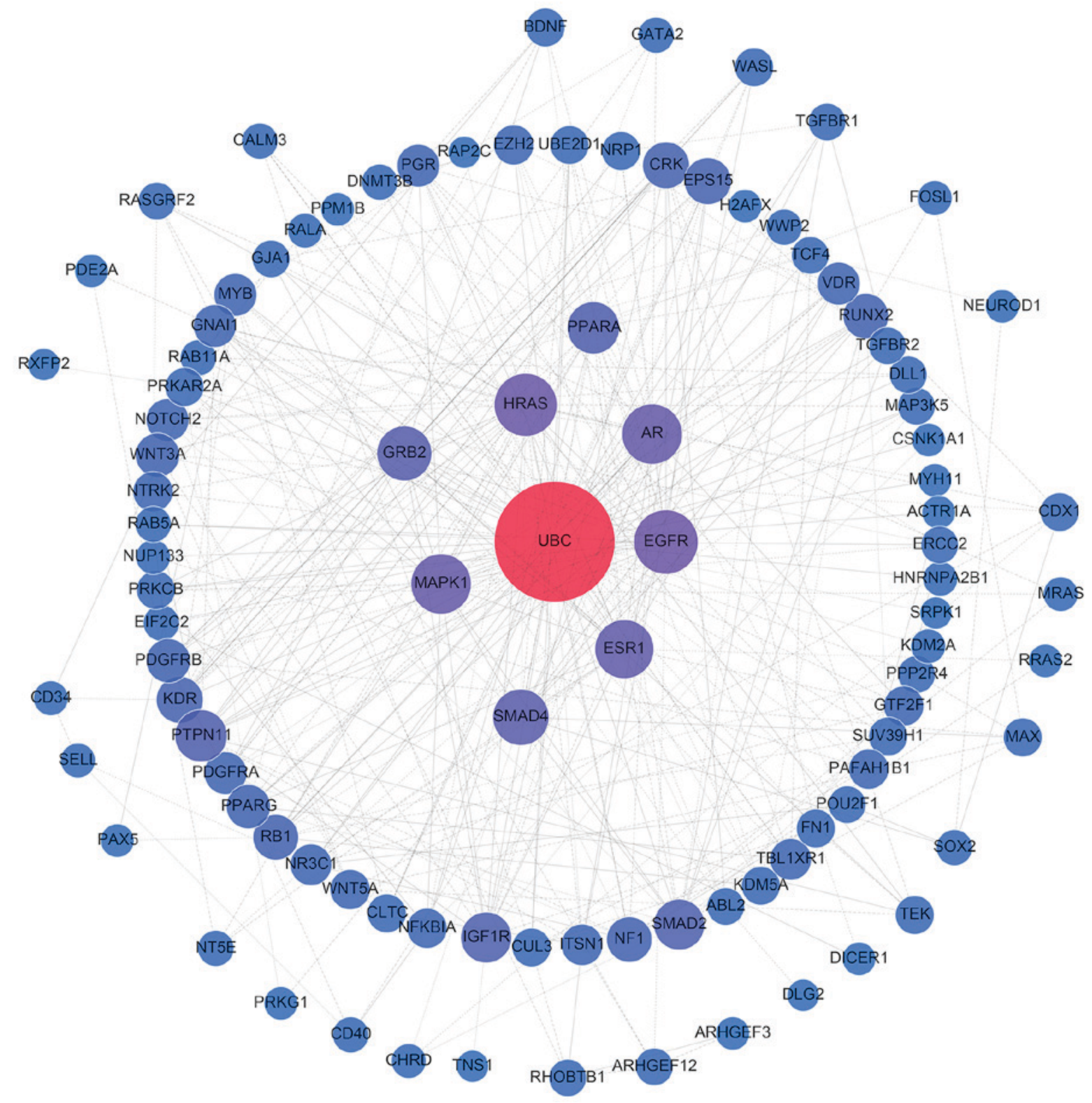

Figure 8. Protein-protein interaction network analysis of the target genes of all 10 miRNAs. Gene interactions were constructed using the STRING online database (string-db.org) and Cytoscape (version 3.4.0; www.cytoscape.org/). Network nodes represent proteins and edges represent protein-protein associations. All target genes of the 10 miRNAs $(\mathrm{n}=1,079)$ were analyzed. miRNA/miR, microRNA.

proliferation. However, a study investigating miR-1301-3p in PAAD is currently not available.

Aberrant activation of the EGFR pathway may suppress tumor progression in retinoblastoma. Zhang et al (38) reported that miR-125a-5p was involved in the EGFR signaling pathway by targeting tafazzin in retinoblastoma. However, there is currently no research involving miR-125a-5p in PAAD. Li et al (39) demonstrated that miR-376b-5p suppressed angiogenesis in cerebral ischemia and halted angiogenesis by targeting hypoxia-inducible factor $1 \alpha$, thereby mediating the vascular endothelial growth factor A/Notch1 signaling pathway. It was identified that miR-328-3p was downregulated in endometrioid endometrial carcinoma, which, was considered an inhibition factor that affected cancer growth and metastasis (40). Leidinger et al (41) also demonstrated that miR-328-5p was upregulated and confirmed to be relevant in lung cancer. Mishra et al (42) identified that miR-454-3p was upregulated in breast cancer, that it may be closely linked to the ErbB signaling pathway and the group also delineated the importance of miR-454-3p as a prospective circulating surrogate molecular signature for the early diagnosis of breast cancer. Recently, Shao et al (43) demonstrated that the survival of those with a high expression of miR-454-3p was poorer when compared with that of those with low miR-454-3p. Wu et al (44) also indicated that BTG anti-proliferation factor 1 was a direct target gene of miR-454-3p. The overexpression of miR-454-3p rendered tumor cells sensitive to radiation (44). In addition, miR-644a-5p has been shown to promote tumor aggressiveness, at least partially, via the downregulation of the Akt/glycogen synthase kinase-3// $\beta$-catenin signaling pathway by targeting paired like homeodomain 2 in esophageal squamous cell carcinoma (45). Chen et al (46) demonstrated that miR-126-3p was associated with advanced pathological stage and lymph node metastasis in patients with lung 


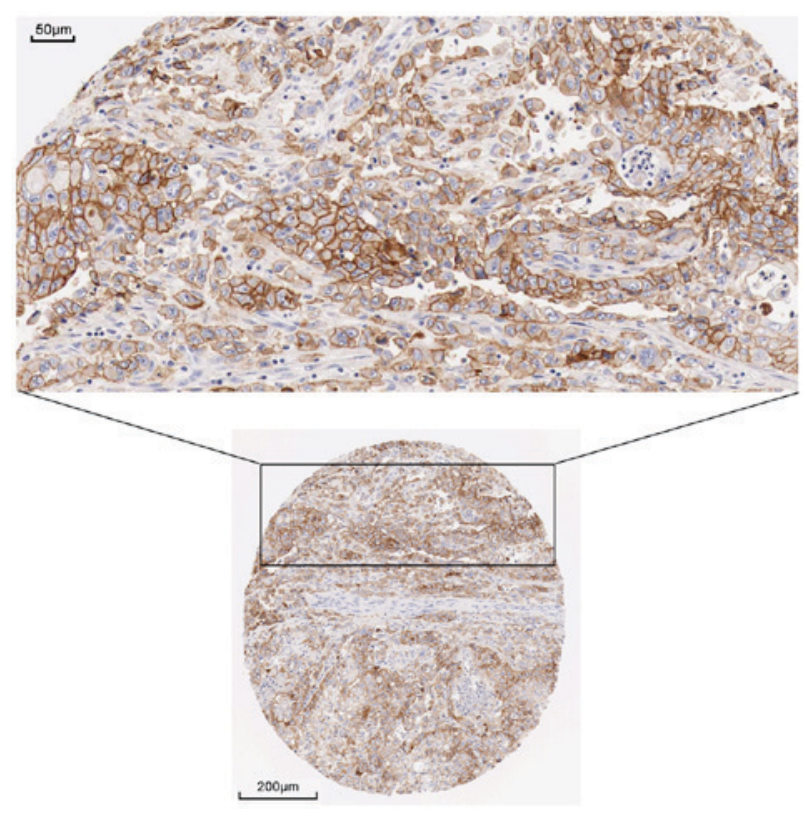

Figure 9. Protein expression of EGFR in pancreatic cancer. The immunohistochemical staining was provided by The Human Protein Atlas. The results revealed a positive expression of EGFR in pancreatic cancer tissues. Scale bars, 50 and $200 \mu \mathrm{m}$, as indicated. EGFR, epidermal growth factor receptor.

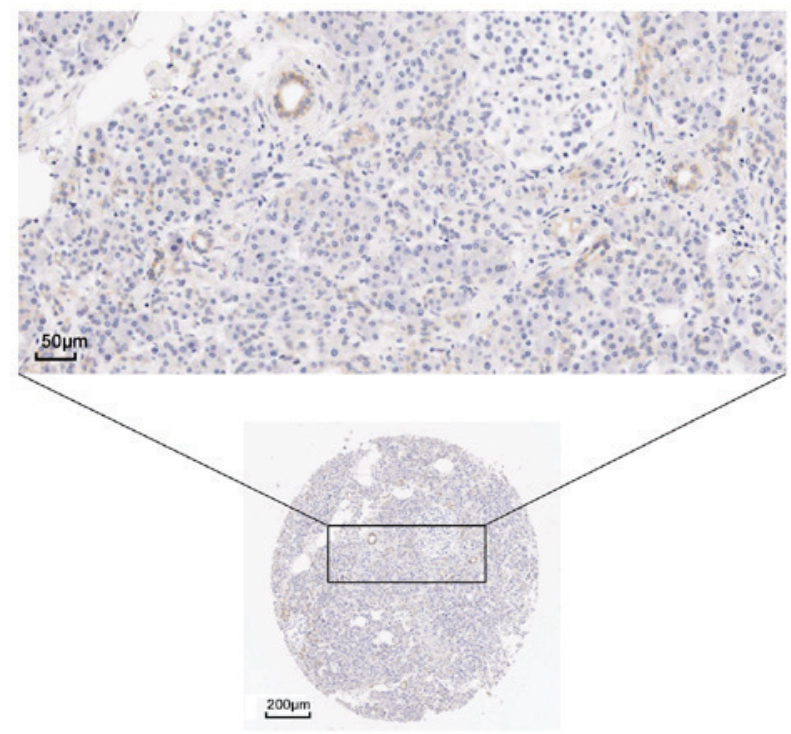

Figure 10. Protein expression of EGFR in normal tissues. The immunohistochemical staining was provided by The Human Protein Atlas. The results revealed a negative expression of EGFR in normal tissues. Scale bars, 50 and $200 \mu \mathrm{m}$, as indicated. EGFR, epidermal growth factor receptor.

adenocarcinoma. miR-126-3p is the most endothelial-specific miRNA that alters vascular integrity and angiogenesis, and it served as a tumor inhibitor targeting phophoinositide-3-kinase regulatory subunit 2 in Kaposi's sarcoma cells (47). Its expression appeared to be upregulated in colon cancer, as demonstrated by Ji et al (48). Furthermore, Suresh et al (49) observed miR-3613-5p enrichment in an extracellular vesicle subtype. Extracellular vesicles were involved in the mechanism associated with the secretion of miRNAs; this enrichment was marked (49). In addition, the miR-29 family, including miR-29a and miR-29b, is well characterized for their ability to regulate extracellular matrix proteins in pancreatic $\beta$ cells (50). Taken together, analysis of TCGA data identified certain miRNAs that were connected with the prognosis of PAAD. As the efficacy of a single biomarker is limited, the pooled signature may be employed instead. Notably, the results of the present study demonstrated that the pooled signature, containing 5 miRNAs, had a greater ability to predict survival. However, the associations between the aforementioned 10 miRNAs and PAAD have not been elucidated. Therefore, the clinical values, as well as the underlying molecular mechanisms, require validation with further studies investigating PAAD.

The present study also attempted to assess the prospective molecular mechanisms of these miRNAs in PAAD. The progression of PAAD tumors is a multi-step process induced by a succession of genetic and epigenetic alterations, that result in the progression of normal cells to cancer cells. miRNAs are involved in this progression by combining the target genes, and then suppressing or decreasing gene expression (51). In the present study, by performing enrichment and function analyses of GO, the target genes of 10 miRNAs were revealed to be involved in a number of crucial biological processes, which, were consistent with developmental processes. From the KEGG pathway analysis, it was concluded that the 'Pathways in cancer' of 'Human Diseases' and 'MAPK signaling pathway' of 'Environmental Information Processing' were the most important signaling pathways in PAAD. The hub genes obtained from the interaction network were assessed in order to comprehensively understand the key role of tumor procession. UBC, SMAD4, MAPK1, AR, ESR1, HRAS and EGFR were identified as hub genes, all of which were shown to participate in 'Signal transduction' of 'Environmental Information Processing'. However, the exact molecular mechanism associated with miRNAs in PAAD requires further study, with in vitro and in vivo experiments.

Recently, Zhou et al (52) reported that a miRNA signature with 13 miRNAs (miR-103-2, miR-125a, miR-126, miR-328, miR-340, miR-361, miR-374b, miR-454, miR-627, miR-664, miR-193b, miR-21 and miR-584) had the potential to be an independent marker for predicting survival in patients with PAAD, which, was also based on TCGA data. Consistent with the results of the present study, miR-125a, miR-328, miR-126 and miR-454 were also identified by Zhou et al (52). However, unlike the present study, Zhou et al (52) did not assess the potential signaling pathways and network of the target genes. The present study conducted bioinformatics analysis of the 10 miRNAs, predicted their target genes and evaluated their roles in the progression of pancreatic cancer. Furthermore, the prognostic information of the patients with pancreatic cancer involved in the present study was updated. In addition, the 5-miRNA signature of the present study had high prognostic significance in clinical practice, and it is more convenient to measure 5 indicators in clinical operations. Also, Zhou et al (52) performed univariate regression analysis in order to investigate their prognostic significance. The 5 miRNAs in the present study differ from those identified by Zhou et al (52) as the prognostic value of each miRNA in the present study was calculated using a multivariate COX regression model which, was adjusted by tumor grade, age and sex.

The present study did have some limitations. Firstly, the miRNA expression data published on TCGA was only collected 
from pancreatic tumor tissues which, may not represent the expression levels in serum or plasma. Furthermore, the sample size of the PAAD TCGA dataset was quite small. In addition, the 10-miRNA signature investigated has not been validated by other detection methods. Although the clinical studies involving the miRNA signature did have significant prognostic value based on TCGA, the functions of the 10 miRNAs are still poorly understood. Thus, well-designed studies are required to investigate the molecular function of these miRNAs in PAAD.

In conclusion, the results of the present study indicated that the 10 miRNAs assessed may be effective markers for predicting the prognosis of PAAD, based on TCGA data. A 5-miRNA signature was also identified which, may be an important predictor of poor survival in patients with PAAD as it exhibited a HR of 0.139 . However, these results require further verification.

\section{Acknowledgements}

The present study was supported by the Fund of Guangxi Natural Science Foundation (grant nos. 2014GXNSFBA118167 and 2016GXNSFBA380039), the Promoting Project of Basic Capacity for University Young and Middle-aged Teachers in Guangxi (grant no. KY2016LX031), the Fund of the Innovation Project of Guangxi Graduate Education (2016) and Natural Science Foundation of China (NSFC 81560448). The authors would also like to acknowledge TCGA Research Network for generating the TCGA datasets (cancergenome.nih.gov/).

\section{References}

1. Yu J, Ohuchida K, Mizumoto K, Fujita H, Nakata K and Tanaka M: MicroRNA miR-17-5p is overexpressed in pancreatic cancer, associated with a poor prognosis, and involved in cancer cell proliferation and invasion. Cancer Biol Ther 10: 748-757, 2010.

2. Wang Z, Li J, Chen X, Duan W, Ma Q and Li X: Disrupting the balance between tumor epithelia and stroma is a possible therapeutic approach for pancreatic cancer. Med Sci Monit 20: 2002-2006, 2014.

3. Liang S, Yang Z, Li D, Miao X, Yang L, Zou Q and Yuan Y: The clinical and pathological significance of Nectin-2 and DDX3 expression in pancreatic ductal adenocarcinomas. Dis Markers 2015: 379568, 2015.

4. Lee SH, Kang CM, Kim H, Hwang HK, Song SY, Seong J, Kim MJ and Lee WJ: Pathological complete remission of pancreatic cancer following neoadjuvant chemoradiation therapy; not the end of battles. Medicine (Baltimore) 94: e2168, 2015.

5. Chen Y, Wang X, Ke N, Mai G and Liu X: Inferior mesenteric vein serves as an alternative guide for transection of the pancreatic body during pancreaticoduodenectomy with concomitant vascular resection: A comparative study evaluating perioperative outcomes. Eur J Med Res 19: 42, 2014.

6. Yang YF, Cao XH, Bao CE and Wan X: Concurrent radiotherapy with oral fluoropyrimidine versus gemcitabine in locally advanced pancreatic cancer: A systematic review and meta-analysis. Onco Targets Ther 8: 3315-3322, 2015.

7. Hezel AF, Kimmelman AC, Stanger BZ, Bardeesy N and Depinho RA: Genetics and biology of pancreatic ductal adenocarcinoma. Genes Dev 20: 1218-1249, 2006.

8. Ali S, Dubaybo H, Brand RE and Sarkar FH: Differential expression of MicroRNAs in tissues and plasma co-exists as a biomarker for pancreatic cancer. J Cancer Sci Ther 7: 336-346, 2015.

9. Loosen SH, Neumann UP, Trautwein C, Roderburg C and Luedde T: Current and future biomarkers for pancreatic adenocarcinoma. Tumour Biol 39: 1010428317692231, 2017.

10. Lewis BP, Burge CB and Bartel DP: Conserved seed pairing, often flanked by adenosines, indicates that thousands of human genes are microRNA targets. Cell 120: 15-20, 2005.
11. Pillai RS: MicroRNA function: Multiple mechanisms for a tiny RNA? RNA 11: 1753-1761, 2005.

12. Xiao J, Peng F, Yu C, Wang M, Li X, Li Z, Jiang J and Sun C: microRNA-137 modulates pancreatic cancer cells tumor growth, invasion and sensitivity to chemotherapy. Int J Clin Exp Pathol 7: 7442-7450, 2014.

13. Lin MS, Chen WC, Huang JX, Gao HJ and Sheng HH: Aberrant expression of microRNAs in serum may identify individuals with pancreatic cancer. Int J Clin Exp Med 7: 5226-5234, 2014.

14. Kai Y, Qiang C, Xinxin P, Miaomiao Z and Kuailu L: Decreased miR-154 expression and its clinical significance in human colorectal cancer. World J Surg Oncol 13: 195, 2015.

15. Hou LJ and Zhai JJ: Aberrant expression profile of translationally controlled tumor protein and tumor-suppressive microRNAs in cervical cancer. J BUON 20: 1504-1509, 2015.

16. Lu Y, Hu J, Sun W, Li S, Deng S and Li M: MiR-29c inhibits cell growth, invasion, and migration of pancreatic cancer by targeting ITGB1. Onco Targets Ther 9: 99-109, 2016.

17. Dong Q, Li C, Che X, Qu J, Fan Y, Li X, Li Y, Wang Q, Liu Y, Yang X and Qu X: MicroRNA-891b is an independent prognostic factor of pancreatic cancer by targeting Cbl-b to suppress the growth of pancreatic cancer cells. Oncotarget 7: 82338-82353, 2016.

18. Li BS, Liu H and Yang WL: Reduced miRNA-218 expression in pancreatic cancer patients as a predictor of poor prognosis. Genet Mol Res 14: 16372-16378, 2015.

19. Brunetti O, Russo A, Scarpa A, Santini D, Reni M, Bittoni A, Azzariti A, Aprile G, Delcuratolo S, Signorile M, et al: MicroRNA in pancreatic adenocarcinoma: Predictive/prognostic biomarkers or therapeutic targets? Oncotarget 6: 23323-23341, 2015.

20. Wang C, Sun Y, Wu H, Yu S, Zhang L, Meng Y, Liu M, Yang H, Liu P, Mao X, et al: Elevated miR-483-3p expression is an early event and indicates poor prognosis in pancreatic ductal adenocarcinoma. Tumour Biol 36: 9447-9456, 2015.

21. BaiZ, Sun J, Wang X, Wang H,Pei H and Zhang Z: MicroRNA-153 is a prognostic marker and inhibits cell migration and invasion by targeting SNAI1 in human pancreatic ductal adenocarcinoma. Oncol Rep 34: 595-602, 2015.

22. Shi H, Chen J, Li Y, Li G, Zhong R, Du D, Meng R, Kong W and $\mathrm{Lu} \mathrm{M}$ : Identification of a six microRNA signature as a novel potential prognostic biomarker in patients with head and neck squamous cell carcinoma. Oncotarget 7: 21579-21590, 2016.

23. Wang M, Wen TF, He LH, Li C, Zhu WJ and Trishul NM: A six-microRNA set as prognostic indicators for bile duct cancer. Int J Clin Exp Med 8: 17261-17270, 2015.

24. Zheng H, Guo X, Tian Q, Li H and Zhu Y: Distinct role of Tim-3 in systemic lupus erythematosus and clear cell renal cell carcinoma. Int J Clin Exp Med 8: 7029-7038, 2015.

25. Dweep H and Gretz N: miRWalk2.0: A comprehensive atlas of microRNA-target interactions. Nat Methods 12: 697, 2015.

26. Kanehisa M, Furumichi M, Tanabe M, Sato Y and Morishima K: KEGG: New perspectives on genomes, pathways, diseases and drugs. Nucleic Acids Res 45: D353-D361, 2017.

27. Zhang SW, Wang J and Pan J: Identification of altered pathways in hypertrophic cardiomyopathy based on combined data of protein-protein interactions and molecular pathways. Genet Mol Res 15, 2016.

28. Jiang M, Zeng Q, Dai S, Liang H, Dai F, Xie X, Lu K and Gao C: Comparative analysis of hepatocellular carcinoma and cirrhosis gene expression profiles. Mol Med Rep 15: 380-386, 2017.

29. Gu Y, Lu L, Wu L, Chen H, Zhu W and He Y: Identification of prognostic genes in kidney renal clear cell carcinoma by RNA-seq data analysis. Mol Med Rep 15: 1661-1667, 2017.

30. Uhlen M, Oksvold P, Fagerberg L, Lundberg E, Jonasson K, Forsberg M, Zwahlen M, Kampf C, Wester K, Hober S, et al: Towards a knowledge-based human protein atlas. Nat Biotechnol 28: 1248-1250, 2010.

31. Uhlén M, Fagerberg L, Hallström BM, Lindskog C, Oksvold P, Mardinoglu A, Sivertsson Å, Kampf C, Sjöstedt E, Asplund A, et al: Proteomics. Tissue-based map of the human proteome. Science 347: 1260419, 2015.

32. Ma MZ, Kong X, Weng MZ, Cheng K, Gong W, Quan ZW and Peng CH: Candidate microRNA biomarkers of pancreatic ductal adenocarcinoma: Meta-analysis, experimental validation and clinical significance. J Exp Clin Cancer Res 32: 71, 2013.

33. Frampton AE, Krell J, Jamieson NB, Gall TM, Giovannetti E, Funel N, Mato Prado M, Krell D, Habib NA, Castellano L, et al: microRNAs with prognostic significance in pancreatic ductal adenocarcinoma: A meta-analysis. Eur J Cancer 51: 1389-1404, 2015. 
34. Cheng PF, Dummer R and Levesque MP: Data mining The Cancer Genome Atlas in the era of precision cancer medicine. Swiss Med Wkly 145: w14183, 2015.

35. Fang L, Yang N, Ma J, Fu Y and Yang GS: microRNA-1301-mediated inhibition of tumorigenesis. Oncol Rep 27: 929-934, 2012.

36. Lin TY, Chen KC, Liu HJ, Liu AJ, Wang KL and Shih CM: MicroRNA-1301-Mediated RanGAP1 Downregulation Induces BCR-ABL nuclear entrapment to enhance imatinib efficacy in chronic myeloid leukemia cells. PLoS One 11: e0156260, 2016.

37. Bi D, Ning H, Liu S, Que $X$ and Ding K: miR-1301 promotes prostate cancer proliferation through directly targeting PPP2R2C. Biomed Pharmacother 81: 25-30, 2016.

38. Zhang $\mathrm{Y}$, Xue C, Zhu X, Zhu X, Xian H and Huang Z: Suppression of microRNA-125a-5p upregulates the TAZ-EGFR signaling pathway and promotes retinoblastoma proliferation. Cell Signal 28: 850-860, 2016.

39. Li LJ, Huang Q, Zhang N, Wang GB and Liu YH: miR-376b-5p regulates angiogenesis in cerebral ischemia. Mol Med Rep 10: $527-535,2014$

40. Xiong H, Li Q, Liu S, Wang F, Xiong Z, Chen J, Chen H, Yang Y, Tan X, Luo Q, et al: Integrated microRNA and mRNA transcriptome sequencing reveals the potential roles of miRNAs in stage I endometrioid endometrial carcinoma. PLoS One 9: e110163, 2014.

41. Leidinger P, Brefort T, Backes C, Krapp M, Galata V, Beier M, Kohlhaas J, Huwer H, Meese E and Keller A: High-throughput qRT-PCR validation of blood microRNAs in non-small cell lung cancer. Oncotarget 7: 4611-4623, 2016.

42. Mishra S, Srivastava AK, Suman S, Kumar V and Shukla Y: Circulating miRNAs revealed as surrogate molecular signatures for the early detection of breast cancer. Cancer Lett 369: 67-75, 2015.

43. Shao N, Wang L, Xue L, Wang R and Lan Q: Plasma miR-454-3p as a potential prognostic indicator in human glioma. Neurol Sci 36 : 309-313, 2015.

44. Wu X, Ding N, Hu W, He J, Xu S, Pei H, Hua J, Zhou G and Wang J: Down-regulation of BTG1 by miR-454-3p enhances cellular radiosensitivity in renal carcinoma cells. Radiat Oncol 9: 179,2014
45. Zhang JX, Chen ZH, Xu Y, Chen JW, Weng HW, Yun M, Zheng ZS, Chen C, Wu BL, Li EM, et al: Downregulation of MicroRNA-644a promotes esophageal squamous cell carcinoma aggressiveness and stem cell-like phenotype via dysregulation of PITX2. Clin Cancer Res 23: 298-310, 2017.

46. Chen Q, Hu H, Jiao D, Yan J, Xu W, Tang X, Chen J and Wang J: miR-126-3p and miR-451a correlate with clinicopathological features of lung adenocarcinoma: The underlying molecular mechanisms. Oncol Rep 36: 909-917, 2016.

47. Wu XJ, Zhao ZF, Kang XJ, Wang HJ, Zhao J and Pu XM MicroRNA-126-3p suppresses cell proliferation by targeting PIK3R2 in Kaposi's sarcoma cells. Oncotarget 7: 36614-36621, 2016.

48. Ji H, Chen M, Greening DW, He W, Rai A, Zhang W and Simpson RJ: Deep sequencing of RNA from three different extracellular vesicle (EV) subtypes released from the human LIM1863 colon cancer cell line uncovers distinct miRNA-enrichment signatures. PLoS One 9: e110314, 2014.

49. Suresh R, Sethi S, Ali S, Giorgadze T and Sarkar FH: Differential expression of microRNAs in papillary thyroid carcinoma and their role in racial disparity. J Cancer Sci Ther 7: 145-154, 2015.

50. Pullen TJ, da Silva Xavier G, Kelsey G and Rutter GA: miR-29a and miR-29b contribute to pancreatic beta-cell-specific silencing of monocarboxylate transporter 1 (Mct1). Mol Cell Biol 31: 3182-3194, 2011

51. Sulpizio S, Franceschini N, Piattelli A, Di Sebastiano P, Innocenti P and Selvaggi F: Cathepsins and pancreatic cancer: The 2012 update. Pancreatology 12: 395-401, 2012.

52. Zhou X, Huang Z, Xu L, Zhu M, Zhang L, Zhang H, Wang X, $\mathrm{Li} \mathrm{H}$, Zhu W, Shu Y and Liu P: A panel of 13-miRNA signature as a potential biomarker for predicting survival in pancreatic cancer. Oncotarget 7: 69616-69624, 2016.

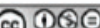

This work is licensed under a Creative Common Attribution-NonCommercial-NoDerivatives 4.0 International (CC BY-NC-ND 4.0) License. 